\title{
Infant Acute Biphenotypic Leukemia
}

National Cancer Institute

\section{Source}

National Cancer Institute. Infant Acute Biphenotypic Leukemia. NCI Thesaurus. Code

C126111.

An acute biphenotypic leukemia that occurs in infancy. 